\title{
Lineamientos de la actualidad epistemológica de la crítica de la economía política en el pensamiento de Marx
}

\section{Guidelines of the epistemological actuality of the critique of political economy in Marx's thought}

\author{
ROBERTO VARGAS MUÑOZ Y PABLO PULGAR MOYA \\ Pontificia Universidad Católica de Valparaiso y Universidad Católica Silva Henriquez
}

Recibido: 14/10/2020 Aceptado: 30/03/2021

\section{RESUMEN}

En este artículo nos proponemos caracterizar algunos de los aspectos centrales de la interpretación marxista contemporánea sobre el método de la crítica de la economía política y los respectivos alcances para (re)pensar la dominación social moderna a partir de la teoría del valor de Karl Marx. Para desarrollar tal objetivo, primero, se expone una revisión crítica de la interpretación marxista clásica sobre sobre la ciencia y el método de Marx, luego, nos adentramos, con esto, en la problemática de la presentación [Darstellung] de la sociedad como formación y realización social del capital arguyendo el vínculo de simultaneidad entre modernidad y capitalismo como relación lógico-conceptual de dominación social.

\section{PALABRAS CLAVE}

MARX, MARXISMO, MÉTODO, DOMINACIÓN SOCIAL, MODERNIDAD

\section{ABSTRACT}

In this article we propose to characterize some of the central aspects of the contemporary Marxist interpretation of the method of criticism of political economy and the respective scope for (re)thinking modern social domination based on Karl Marx's theory of value. To develop this objective, first, a critical review of the classical Marxist interpretation of Marx's science and method is exposed, then, with this, we delve into the problem of the presentation [Darstellung]

\footnotetext{
(C) Contrastes. Revista Internacional de Filosofía, vol. XXVI N³ (2021), pp. 111-132. ISSN: 1136-4076

Departamento de Filosofía, Universidad de Málaga, Facultad de Filosofía y Letras Campus de Teatinos, E-29071 Málaga (España)
} 
of society as formation and social realization of capital arguing the link of simultaneity between modernity and capitalism as a logical-conceptual relationship of social domination. KEYWORDS

MARX, MARXISM, METHOD, SOCIAL DOMINATION, MODERNITY

\section{INTRODUCCIÓN}

¿POR QUÉ CADA CIERTO TIEMPO reaparece insistentemente el espectro de Marx? Según el célebre pensador comunista español, Manuel Sacristán, los clásicos al interior de las ciencias sociales se caracterizan por ser autores irrenunciables $\mathrm{y}$, hasta cierto punto intempestivos, mas, a la vez, no actualizables en todos los detalles que le secundan. ${ }^{1}$ El carácter irrenunciable del proyecto marxiano, probablemente, estriba en la forma que adopta el análisis: la crítica de la economía política se yergue como crítica del capitalismo, de la sociedad burguesa y de la modernidad, caracterización tríadica de un mismo objeto de estudio, el cual se torna imposible de agotar en la exposición genética alguna de la economía, en la geografía económica inglesa del siglo XIX, en la expresión de lucha de clases propia del siglo XX o en las transformaciones geopolíticas del multipolarismo del siglo XXI. Una de las razones de tal peculiaridad ha de encontrarse en los pasajes del tomo III de El Capital, en la medida que Marx explica que su preocupación primera giraba en torno al modo de producción capitalista en un término ideal. ${ }^{2}$

Para proceder a una investigación del tal magnitud, Marx debía, como indica un ya clásico como Roman Rosdolsky, repensar en «[e]l papel de la abstracción en la economía política» (Rosdolsky 2004 [1968], p.615). Según este autor, primer comentador de los Grundrisse, este papel exhibe un problema metodológico respecto a términos robustos como lo serán forma [Form] y contenido [Inhalt]. En efecto, las formas burguesas de producción aparecen a los economistas clásicos como elementos naturales e inalterables, problemática que Marx lee como un marcado sesgo epistemológico al interno de las reflexiones analíticas, sobre todo al interno de los reputados enunciados de Adam Smith y David Ricardo en torno a la caracterización de la riqueza. Según Rosdolsky, aquel punto de inflexión les inhabilita para desarrollar genéticamente el problema de las formas sociales estrictamente modernas. En cambio, estas mismas formas llegan a ser consideradas puramente en su aspecto formal, ratificando una supuesta - o superpuesta - coincidencia con un contenido a histórico.

1 Cf. Sacristán 2003, p.181.

2 Cf. Marx 2012, p.839. 
Siguiendo a Lukács, Rosdolsky considera que lo «no-dialéctico» de los análisis teóricos de Ricardo y de los clásicos en general, mienta una elusión de las categorías de mediación y, por ende, una derivación de los fenómenos de la superficie económica a partir de abstracciones meramente formales y simples. Las «categorías mediadoras», nos comenta Rosdolsky, o también llamadas «transiciones», componen los «eslabones intermedios» para la metodología marxiana (Ibid., p.618) y, por extensión, podríamos agregar aquí, los pilares de los supuestos epistemológicos de Marx.

Las consideraciones marxianas acerca de lo innecesario de la ciencia como coincidencia entre la forma de manifestación -tal como se muestra en la superficie- y la esencia de las cosas -figura medular interior- no es utilizada en Rosdolsky solo para criticar y actualizar las diferencias entre Marx y los clásicos, los cuales, como señala, descuidan la forma al darla en sacrificio en mor del contenido. También señala que hay una tendencia a absolutizar las formas, práctica teórica que ubica en la crítica moderna del capitalismo llevada a cabo en la experiencia socialista soviética cuando «deducen del sustrato suprahistórico de la determinación del valor el carácter suprahistórico de la forma valor ¡Como se ve, la significación metodológica de la dialéctica para la economía marxista nunca podrá estimarse suficientemente!» (Ibid., p.617). Como veremos en el siguiente apartado, podemos encontrar una explicación tentativa a la crítica esbozada por Rosdolsky en las lecturas de los marxistas clásicos sobre la ciencia en Marx, las cuales lejos de mentar interpretaciones hermenéuticas o rayanas a una «marxología», se trataron de exégesis con alta significación y preponderancia política, en tanto que encontraron en la obra de Marx y Engels no solo un aparato crítico categorial para desarrollar un análisis del modo de producción capitalista, sino que también una legitimidad política y epistémica, al localizar en el «marxismo de Marx» una cierta ciencia del proletariado.

En el presente artículo proponemos caracterizar y sistematizar algunos de los aspectos centrales de la interpretación marxista clásica sobre los conceptos de ciencia y método en Marx, identificando críticamente los argumentos que llevan a concebir al marxismo como una ciencia positiva con tres fuentes únicas. Posteriormente, presentamos los debates contemporáneos sobre el método de la crítica de la economía política y los alcances para (re)pensar la dominación [Herrschaft] social moderna a partir de la teoría del valor. Sostenemos que una de las vías para sostener el vínculo de simultaneidad entre modernidad y capitalismo desde la crítica de la economía política es a través de una relación lógica entre método y dominación social desde donde es posible comprender cómo el capitalismo se declina de tal manera que encuentra en la modernidad la vía de realización del capital. Planteamos que una teoría de la dominación social en Marx critica del capitalismo y de la 
modernidad debe necesariamente, superar el concepto de ciencia y método tradicionales del marxismo clásico («ideológico»; del «movimiento obrero») y, reinterpretar el uso de la abstracción en Marx y, por tanto, el método de la crítica de la economía política, como algo no separado de su objeto de la formación social [Gesellschaftsformation] en el capitalismo y la modernidad. Esto se puede encontrar en el Marx tardío (sobre todo en los Grundrisse y Capital) y en un dialogo con las nuevas lecturas de Marx y los encuentros conceptuales con la lógica de Hegel.

En el siguiente apartado caracterizaremos el problema de la recepción del concepto de ciencia en Marx. Nos interesa mostrar que en tal ejercicio, la recepción canónica de Marx no incorpora (interpreta) un concepto de ciencia ni de método (en Marx) para pensar la dominación social moderna bajo un carácter abstracto. Consideramos que esto separa a Marx no solo del marxismo clásico, sino también de la economía política del siglo XIX y de la «corriente principal» en economía o de cualquier registro disciplinar particular. Marx pone como objeto la «moderna sociedad burguesa» cuestión que no agota el saber de una ciencia disciplinar (como ciencia particular-dificultad, creemos, del marxismo tradicional), problemática que le exige construir un método para su investigación que sea uno con su objeto. Tal proposición, evidentemente, nos remite a las nuevas lecturas sobre la relación Hegel-Marx, no obstante, un diálogo con obras canónicas como la Ciencia de la Lógica, se transforma en una tarea que desborda el próposito de este escrito. Como veremos en el apartado tres, el problema del marxismo tradicional respecto de la ciencia también se expresa en la manera en cómo se concibe el método. Tales problemas evidenciados exigen una vinculación interpretativa de la teoría del valor a fin de pensar la dominación social moderna bajo su impronta lógica, en la medida que su presentación u exposición [Darstellung] requiere una especial atención en tanto que es ésta la que despliega explicativamente las formas sociales (abstractas) dominantes de la modernidad capitalista.

\section{BuSCAR LAS FUENTES DEL PENSAMIENTO EN MARX}

La interpretación marxista clásica de la ciencia marxiana ${ }^{3}$ derivó en lo que, a día de hoy, podríamos denominar como «tradición del marxismo científico», con la cual, el marxismo en general, tuvo que lidiar, ya sea en su defensa o crítica. Es posible cartografiar el origen de tal visión en la propia

3 A modo de diferenciación y siguiendo la nomenclatura regular, vamos a reservar, por un lado, el término «marxiano» para aquellos aspectos que denoten un vínculo necesario al pensamiento del propio Marx. Por otro lado, enfatizaremos con el vocablo «marxista», aquellas posiciones teóricas o políticas, in sensu lato, de que toman a Marx como base. 
lectura del Engels maduro, especialmente aquel de Anti-Dühring de 1878, obra en donde se da cuenta de una distinción insalvable entre materialismo e idealismo y, a su vez, entre metafísica y dialéctica, retomando hipótesis formuladas embrionariamente en Ideología alemana. Efectivamente, para Engels, el corpus de pensamiento que comparte con Marx tiene de suyo un carácter científico en tanto que demostraba determinadas leyes inmanentes del despliegue epistémico de la sociedad y de la naturaleza, una tal operación constituiría, del mismo modo, una equivalencia entre ciencia social y ciencia natural, la cual será desarrollada posteriormente por la archiconocida corriente del marxismo -leninismo ${ }^{4}$.

El tránsito de la nomenclatura socialista hacia un carácter de ciencia [zur Wissenschaft ] adscrita por Engels (1962b) se distinguía, a su modo de ver, de modo contrapuesto de cualquier propuesta de socialismo utópico y tenía como intención primera la demarcación del abismo epistémico entre utopía y ciencia, para, de esta forma, consolidar una concepción de la sociedad basada en leyes y en un método certero e infalible. Según Engels, la «noción materialista de la historia» (Ibid., 209) y el descubrimiento del secreto de la plusvalía, son los hallazgos cruciales que transforman a la teoría socialista moderna en ciencia, con una radical diferencia de las tratativas de Saint Simon, Fourier y Owen, bastiones de la lectura «utópica». Sin embargo, podríamos agregar, en este respecto, que no sólo tenía una pretensión de distanciamiento del llamado socialismo utópico, sino que también con otras variantes de la época muy en boga, como el «socialismo revolucionario» del siglo XVIII, los «socialistas reformistas o socialistas de Estado» (Blanc, Rodbertus, Lasalle) o los «socialistas ricardianos» (Hall, Hodgskin, Thompson, Gray, Bray).

$\mathrm{La}$ argumentación de Engels del rótulo «utópico» a esta propuesta venía justificada mediante tres acontecimientos troncales: i) el hecho que a mediados del siglo XVIII el proletariado apenas empezaba a destacarse en el seno de las masas desposeídas; ii) el modo de producción capitalista $\mathrm{y}$, con ello el «antagonismo» entre burguesía y proletariado, solo se había desarrollado escasamente $y$, finalmente, iii) el hecho que las teorías de este tipo de socialismo habrían reflejado un estado rudimentario e inicial del modo dominante de la producción capitalista posterior y, por tanto, de la condición de clase que genera. En resumen, el carácter incipiente del tiempo histórico habría condenado a estos pensadores a moverse en un rango de especulación fuera de condiciones históricas que permitieran un análisis material de la totalidad social, por tanto. Según Engels ${ }^{5}$ la condición acelerada de su propio tiempo histórico le permitían tanto a él, como a Marx, empero, racionalizar el

4 Cf. Löwy 1975, pp.198-202.

5 Cf., Ibid, p. 197. 
proceso de la moderna «formación social» [Gesellschaftsformation] de manera científica, pues la lucha de clases emprendida entre burguesía y proletariado adquiría un carácter constituyente de la propia historia de los países europeos avanzados, esto junto a un desarrollo precipitado de la industria y con ello la dominación política de la burguesía, permitiendo, por ende, comprender de modo retroactivo que el desarrollo histórico precedente como forma una inmanente pugna entre clases con poder asimétrico, frutos de relaciones de producción e intercambio.

Esta propuesta engelsiana, fue posteriormente recuperada, recogida y sistematizada por Kautsky en Las tres fuentes del marxismo de 1907, texto en el que caracterizaba al marxismo como ciencia de la sociedad (desde la perspectiva del proletariado) y como óptima síntesis del pensamiento alemán, francés e inglés. Esta último descripción cumplía un doble objetivo, por un lado, alentar el internacionalismo entre los miembros de la II Internacional, de la cual Kautsky era su presidente, y, por otro, hacer del marxismo una teoría del proletariado, la «síntesis» de las «mejores» expresiones teóricas de la historia europea $\mathrm{y}$, por tanto, pretendía obtener una validación axiológica, histórica e intelectual, en tanto criterio externo de validación. Consecutivamente, siguiendo a Kautsky, Lenin publica Tres fuentes y tres partes integrantes del marxismo en 1913, un breve artículo donde presenta al marxismo como una teoría que bebía y se cristalizada de tan solo tres fuentes nutrientes.

La propuesta de Lenin se podría resumir de la siguiente manera: i) la primera fuente sería el pensamiento alemán, a través de la «dialéctica» de Hegel y del «materialismo» de Feuerbach, como unicidad constitutiva de un corpus teórico inicial: el así llamado «materialismo histórico»; ii) la segunda, la economía política inglesa, la cual sentaba las bases, a través de los trabajos de Smith y Ricardo de la teoría del trabajo como base de todo valor, conformando con ello una segunda característica del marxismo: «la teoría de la plusvalía»; por último, iii) la tercera fuente, el socialismo francés, utópico de Cabet, Fourier, Owen y Saint Simon, que a partir de sus intuiciones era factible reformular, científicamente, «la doctrina de la lucha de clases». Según Lenin, sin estas tres fuentes no habría sido posible para Marx desarrollar y profundizar el carácter científico de su propio pensamiento y, por ende, una propuesta criteriosa en mor del término de las relaciones de dominio efectivo sobre la masa proletaria.

Según Balibar, este artículo de Lenin cumple un rol desdoblado muy similar a la intención kautskyana, es decir, tanto promocionar el internacionalismo, como hacer del marxismo «la teoría del proletariado como una totalización de la historia europea que instituye el reino de lo universal» (Balibar 2000, p.10ss). En 1841 Moses Hess en La triarquía europea ya había expuesto una teoría «del proletariado», dando cuenta, que ésta ya era 
una idea latente que rondaba Europa y que el propio Marx había recogido en sus escritos de juventud. En 1990 Costanzo Preve abrió nuevamente el debate en torno a las fuentes, presentando cuatro maestros de Marx: Epicuro (materialismo de la libertad), Rousseau (democratismo igualitario), Smith (el trabajo como fundamento de la propiedad), Hegel (contradicción dialéctica e historicidad). Sin embargo, Preve desecha a la llamada corriente utópica como fuente de inspiración para un (joven) Marx, desatendiendo su rol tanto en la conformación epistémica de Marx, como en las consecuencias de esta corriente en las formulaciones (críticas) del socialismo soviético y del Este hicieran de su propio marxismo.

Por supuesto, el problema de la interpretación de fuentes va mucho más allá de la nomenclatura esbozada por el marxismo clásico. La crítica vertida contra la tipología interpretativa dominante desde pensadores contemporáneos a complejizado la misma problemática de las «fuentes» en una cuestión no solo vinculada a la caza de «fuentes» directas, sino que se ha ampliado hacia campos hermenéuticos, filológicos, literarios, etc. David Harvey (2014, p.10) es de la opinión que, para una interpretación detallada y profusa de la obra magna de Marx, El Capital, se requiere de una actitud abierta que solo se emprende mediante la consideración de las propias lecturas literarias de Marx, las cuales permiten en muchas ocasiones dar cuenta del uso metafórico, irónico o, incluso, poético de algunos enunciados. ${ }^{6}$ Francisco Fernández Buey y Ludovico Silva han enfatizado el vasto y erudito dominio idiomático de Marx, el cual fue clave para el acceso, de primera fuente, de textos y manuscritos que de otra manera permanecían ocultos. ${ }^{7}$ Algunos investigadores contemporáneos han ampliado el debate en torno a las fuentes tanto en el ámbito de la filosofía, como en el de la economía. Nicolás González Varela pesquisa sobre la recepción de Spinoza en Marx a lo largo de su obra concluyendo que «la filosofía política de Marx, los alcances éticos y políticos» (Varela 2012, p.8) no podrían ser entendidos sin una comparación hermenéutica con la obra de este filósofo. ${ }^{8}$ Diego Guerrero (2011), por otro lado, mediante un detallado análisis económico ubica como

6 No solo Harvey identifica como primordial un repaso por literatos que se repiten de manera constante en la obra de Marx, entre los que se cuentan Lessing, Dante, Homero, Shakespeare, Balzac, Shelley, Heine o Goethe, sino que también autores como Heinrich (2018, p.43ss.), Çidam (2012) o Jappe (2016, p.154) enfatizan en la influencia del Fausto en la construcción de «sujeto automático» al interior de la teoría del valor del Capital.

7 Cf. Fernández Buey 1984, pp.35ss: Silva 1971 pp.15-27. El dominio del francés, inglés, griego, latín, italiano y ruso para leer y empaparse del estilo literario

8 El rodeo en torno a Spinoza esbozada con fuerza por Althusser (cf. p.ej. Althusser 1974, p.73s; 2014, p.276ss) ha sido retomado en innumerables ocasiones, sea como intento hemenéutico, sea a modo de interludio filosófico o bien a modo de propuesta alternativa a un dominante hegelomarxismo del siglo XX en el ejercicio interpretativo de la totalidad social. Algunos ejemplos 
fuente indiscutible - sin negar con ello la preponderancia de David Ricardo - a Sismondi, localizándolo como autor clave para la formulación madura de Marx sobre el fetichismo de la mercancía.

En efecto, la diversidad de las fuentes que nutren la producción teórica marxiana sobrepasa largamente los mencionados tres registros clásicos del marxismo. Este breve acápite, ha de patentar que la construcción de una «ciencia»se trataría, por ende, de la construcción de una crítica categorial que funciona como una crítica práctica de la realidad, es decir, de un proyecto crítico para socavar las certidumbres de la época definidas en el proyecto liberal de los economistas y filósofos del siglo XVIII y XIX.

\section{PRESENTAR EL MÉTODO}

La interpretación de Engels sobre la cienciay, en particular, sobre el llamado materialismo histórico, constituyó una clave fundamental para la comprensión del marxismo tradicional. De la misma forma, la interpretación engelsiana sobre la composición metodológica del Capital tuvo consecuencias relevantes para la conformación del marxismo anteriormente señalado. Para Engels, el corpus de pensamiento no puede interpretarse correlativamente como «una doctrina, sino [como] un método. No ofrece dogmas hechos, sino puntos de partida para la ulterior investigación y, el método para dicha investigación» (Engels 1976 [1895], p.534). Engels, en defensa de una concepción sobre el método en tanto dialéctico, fijó los principios de una dialéctica entendida como ciencia que denota leyes de movimiento y desarrollo [Bewegungs- und Entwicklungsgesetzen] coherentes y concatenados entre naturaleza, sociedad humana y pensamiento (Engels 1962b, p.131s). Esta interpretación se volvió rápidamente en canónica instituyendo, de esta manera, los cimientos de lo que posteriormente se denominará «materialismo dialéctico» ${ }^{9}$, en tanto «descubrimiento» de leyes inmanentes que rigen tanto procesos de la historia como de la naturaleza y de su concordancia con el carácter escéptico del propio pensar. ${ }^{10}$

notables: cf. Breton 1982-3, pp.61ss; Macherey 1997, p.184; 2014, 31ss; Balibar 2011; 2000, p.36s; Casanova 2007, p.361ss; Morfino 2014, pp.13-45 y pp.113-131.

9 Respecto al mentado «materialismo dialéctico»o «carácter dialéctico de la naturaleza» se han ya vertido ríos insondables de tinta. La noción será incorporada a la teoría política en 1887 por Josef Dietzgen y sistemáticamente desarrollada por Plejanov y Lenin (Sobre ello, cf. Ruiz Sanjuán 2014, p.145s). Damos acá algunas referencias clásicas que pueden ser aclaratorias sobre una comprensión crítica de esta dialéctica, cf. Bobbio 1999, p.110; Zelený 1968, Fink-Eitel 1987, p.164ss o Mark Rosental 1969.

10 No obstante, lo determinante de la posición de Engels respecto de la comprensión del método en Marx es que interpreta críticamente al método en tanto «histórico-lógico», donde el orden lógico se manifiesta como reflejo abstracto del mismo proceso histórico. Para Engels «la 
Siguiendo estos aspectos, planteó Oskar Lange (1966, p.94) que el método «se descompone en tres procedimientos sucesivos de investigación: la abstracción, la concretización progresiva y la verificación» siendo El Capital un «ejemplo de concretización progresiva» (Ibid., p.108). Sin embargo, discutiendo con Lange, Rosdolsky consideró que «lo que falta en el capítulo metodológico de éste es precisamente el 'alma' del método político económico de Marx: ¡su dialéctica!» (Rosdolsky 2004 [1968], p.615). Y efectivamente, fue él quien propugnara una re-lectura del Capital a la base de los Grundrisse, acentuando la influencia hegeliana en Marx y concentrando la atención en la abstracción como momento esencial del proyecto de la crítica de la economía política. La discusión contemporánea sobre el método ha abierto nuevos canales y vínculos entre la obra de Marx y Hegel para cuestiones de orden epistémicas y metodológicas inscritas en el proyecto de la crítica de la economía política de Marx.

Sobre la actualidad y los laberintos de la obra de Marx, a lo largo de las últimas décadas, diversas discusiones tanto sobre el «carácter lógicosistemático», como el «método dialéctico» y la «estructura del movimiento epistemológico» han sido emprendidas desde fines del siglo XIX. Después de Lenin, Rubin, autores como Zelený, Fink-Eitel y Mark Rosental presentaron formulaciones metodológicas detalladas en la primera mitad del siglo XX. Sin embargo, en la actualidad podemos presenciar la así llamada «disputa por el método» [Methodenstreit], que cuestiona el contexto en el que emergen y son consideradas las formas del valor. La controversia entre Haug y Heinrich ha proporcionado hasta ahora uno de los argumentos epistemológicos más enriquecedores sobre el concepto de método en Marx e ilustrado nuevas rutas de investigación. La argumentación de Heinrich y la, así llamada, Neue-MarxLektüre ha ejercido una crítica enérgica a la lectura lógico-histórica del objeto metodológico de investigación de Wolfgang Fritz Haug y de Klaus Holzkamp enfatizando que «la presentación del proceso histórico de formación de la totalidad está más allá del desarrollo dialéctico de las categorías» (Heinrich 1991 ${ }^{1}$, p.177). Sin querer profundizar demasiado en estas primeras líneas, es necesario mencionar que esta polémica - hasta cierto punto dicotómica - se arrastra sustancialmente hasta nuestros días. ${ }^{11}$

Consideramos que en la presentación o exposición [Darstellung]

crítica de la economía política podía construirse de dos modos: el histórico o el lógico» (Engels $1974^{9}$, p.128). Si por un lado, el modo histórico «sigue el desarrollo real de las cosas», incorpora este, empero, el riesgo de romper las ilaciones lógicas que le dotan de sentido. En este respecto, aparece el método lógico, por otro lado, como la indicada exposición despojado de las formas históricas que la componen y de la contingencia inmanente de la historia.

11 Las controversias de investigación más conocidas en los últimos años también incluyen la relación específica entre la forma del valor, la génesis del dinero y el pro- 
metodológica de la sociedad burguesa en Marx, se juegan algunos de los aspectos fundamentales de la actualidad epistemológica de la crítica del capitalismo y la crítica de la modernidad y de la simultaneidad de ambos. ${ }^{12}$

En el contexto de la discusión marxista sobre el método, el término «formación social» [Gesellschaftsformation] acuñado por Marx se toma en cuenta como un concepto central. Para tal análisis subyace, sin embargo, una dificultad inmanente, en la medida que, para Marx, la formación social tiene realidad solo en tanto totalidad de la crítica de la economía política, lo que se pone en evidencia cuando se considera que el desarrollo conceptual de las diversas formas económicas tiene existencia efectiva precisamente por medio del desarrollo o la formación de la sociedad. De este modo, Marx conceptualiza el término «formación social» como una voz para determinar la organización genética de diferentes etapas históricas, como lo serán expresiones de socialización tan diversas como comunismo primitivo, feudalismo, capitalismo, etc. ${ }^{13}$ En suma, para Marx, las diferentes épocas de la historia mundial pueden llegar a ser caracterizadas como tal solo según la expresión de sus diferentes formas sociales. Para profundizar en la comprensión de estas formas es necesario, empero, explorar la forma determinada de su enriquecimiento, o lo que es lo mismo, la forma específica de desarrollo de sus relaciones de producción.

Los fundamentos de la teoría de la formación social entienden la historia de las relaciones económicas como un desarrollo progresivo de su forma de riqueza, o dicho sintéticamente, de su forma-riqueza. Por lo tanto, toda comprensión de las relaciones fundamentales de una determinada formación social debe ir precedida de una comprensión cabal y orgánica de momentos y determinaciones de todas las relaciones de producción, las cuales performan de modo particular la riqueza. El análisis histórico-filosófico de la formación de la riqueza define, por ende, la formación social. Es a través del desarrollo histórico de la riqueza donde se forma la esencia de una sociedad específica, que a su vez puede diferenciarse de otras formas de la sociedad en mor de la presentación no-empírica de la producción de riqueza. ${ }^{14}$

La investigación sistemática del modo de presentación metódico del capital en la reconstrucción de la sociedad burguesa de Marx tiene una relevancia

ceso de intercambio y la «reducción dialéctica». Algunos debates pueden encontrarse ilustrados en: cf. Hoff 2009, p.95; Riedel 1997, pp.3-40; Reichelt 2000, pp.100-126.

12 Cf. Pulgar 2017, p.355s.

13 Utilizamos el término de Adorno «socialización» [Vergesellschaftung] como sinónimo de «formación social», en la medida en que esto también describe el proceso de formación y desarrollo de una forma de sociedad.

14 Diferentes formas de riqueza pueden coexistir en una época común. Pero lo que define una era es el dominio efectivo de un modo de producción sobre todos los demás. 
metodológica y de desarrollo del llamado relato marxiano de la formación social moderna, un problema que tuvo un gran impacto en la disputa por el método en Marx de los últimos treinta años en la literatura en lengua alemana y extendido a especialistas dentro del mundo anglosajón e italiano.

\section{HEGEL Y EL MÉTODO DE LA CRÍTICA DE LA ECONOMÍA POLÍTICA ${ }^{15}$}

El pensamiento de Marx se desarrolla, desde enfoques tempranos, teóricamente inmaduros, hacia puntos de análisis familiarizados con un desarrollo sistemático y un análisis detallado del modo de interacción económico-político al interior la sociedad burguesa. Sin embargo, ante la pregunta sobre la modalidad de este modo de interacción, se nos hace ineludible, entonces, la pregunta por un posible, factible o necesario examen en torno la presentación/exposición metodológica, dando con el primer relato en la misma exposición crítica del pensar de la Ciencia de la lógica, en tanto antecedente inmediato sobre la discusión epistemológica.

Es lugar común tanto la afirmación como la negación de que Marx y Hegel contribuyeron por caminos disímiles, pero en ciertos puntos consonantes, al desarrollo del método sistemático de presentación crítica. Aunque bien, Marx solo escribió motu proprio brevemente en sus pocos escritos metodológicos explícitamente sobre este «modo» de «presentación crítica», éstas se encuentran entre las reflexiones más polémicas para el debate contemporáneo en torno a la problemática del método. La inmanente unidad de los conceptos de crítica y presentación, se puede encontrar de una manera análoga o bien similar en la autopresentación [Selbstdarstellung] epistemológica de Hegel del pensar puro decantado en el concepto ${ }^{16}$. El método especulativo de Hegel demuestra ser particularmente útil para aclarar ciertas fortalezas terminológicas de la crítica sistemática de la sociedad burguesa descrita en El Capital. Un diálogo crítico con la filosofía de Hegel, sobre todo con su Lógica, permite que nuestro análisis ponga en contraposición el carácter lógico, que pone bajo la lupa la matriz histórica (no historiográfica) de las relaciones sociales y que aparece como sistematizante de la paleta de categorías y conceptos de él derivados. ${ }^{17}$

La cuestión metodológica del desarrollo de la presentación crítica en la obra tardía de Marx nos permite definir las diferentes manifestaciones como elementos fundamentales de la reproducción de la sociedad capitalista.

15 En este apartado no tiene por objeto reconstruir la relación Hegel-Marx, más bien, mostrar algunos de los vínculos relevantes sobre tal relación y cómo han permitido pensar el problema del método para re-pensar la teoría del valor marxiana como base de la dominación social abstracta moderna.

16 Cf. Hegel, G.W.F. 1986, § 160, p. 307

17 Cf. Schäfer 2019; Pulgar \& Narváez 2020. 
El «modo de presentación crítica»o, lisa y llanamente, el «método» de la economía política, entendido acá como una forma de justificación científica, es interpretado y descrito de maneras muy diversas por la doxografía marxista: como una "presentación dialéctica» ${ }^{18}$, como una «presentación dialécticasistemática» ${ }^{19}$, como una «presentación científica» ${ }^{20}$, como una «presentación inmanente ${ }^{21}$ o como una «presentación metodológica» ${ }^{22}$, entre otras. Estas diferentes nomenclaturas funcionan enfáticamente como una caracterización determinada de la «presentación»o «exposición», pero ellas, en el ejercicio de contraposición, manifiestan marcadas diferencias epistemológicas, precisamente porque conceptos como «crítico», «dialéctico», «metódico»o «científico» no están inmediatamente directamente relacionados.

La cuestión de la presentación o exposición metódica es una de las más relevantes en el examen en torno al método de Marx. La caracterización unificada de la presentación como una crítica de la formación social burguesa se vuelve un punto de vista clave para interpretar la posibilidad de un método. La crítica orientada a la presentación de la economía política en el Capital contiene un carácter correctivo con respecto a las manifestaciones, no directamente en la forma de desenmascaramiento, sino en la forma de una reconstrucción conceptual de la socialización gradual. De esta manera, el carácter escéptico en la presentación se torna necesario a fin de comprender la génesis de los niveles programáticos de abstracción en su obra.

Así, la explicación genético-conceptual, el examen sistemático de la presentación y de su método subyacente se yergue como una de las tareas principales de la investigación contemporánea sobre el Marx, por lo menos, tardío. Será en el copiosamente citado epílogo de la segunda edición del Capital, donde Marx insistirá en una distinción programática entre modo de presentación y modo de investigación. ${ }^{23} \mathrm{Si}$ bien el modo de investigación consiste en una investigación empírica, histórica y analítica, el modo de presentación es la formación teórica conceptual y la clasificación del «material» explorado en las manifestaciones.

La formación de la moderna sociedad burguesa no se refleja en el trabajo tardío en un sentido histórico cronológico, sino como una sistematización abstracta. La presentación crítica de Marx tiene en común con la presentación científica en la Ciencia de la Lógica de Hegel, en este respecto, que ambos

18 Cf. Heinrich $1991^{1}$, p.144-151; Henning 2005, pp.145, Arthur 2004, pp.79, Wolf 2006, pp.159-188.

19 Cf. Reuten \& Williams 1989; Gallas 2005, p.403.

20 Cf. Wolf, D 2004, pp.23, 35ss; Elbe 2010.

21 Cf. Arndt $1985^{1}$, pp.9ss; Brentel 1989, pp.301s.

22 Cf. Wolf, D 2004.

23 Cf. Marx 1972, p.27 
deben caracterizarse como desenlazados en la exposición de su carácter empírico. Una diferencia importante del relato marxista no convencional a la mayoría de los otros enfoques de la economía política moderna, como por ejemplo, la teoría laboral del valor de Ricardo ${ }^{24}$ consiste en el carácter dialéctico-sistemático, en el que la clave para la comprensión correcta y adecuada de la economía reside en la combinación de todo el conocimiento relevante sobre una sociedad total. La «presentación de la sociedad» es, en consecuencia, la manifestación concatenada de una totalidad concreta. A día de hoy, esta unificada totalidad social formal encontrará nombre bajo el término «capitalismo», «modernidad» o, bien, «hegemonía del capital».

Es necesario determinar aquellos límites metodológicos abstractos que se generan/son generados de la unidad entre crítica y presentación. En este contexto, el concepto de valor debe ser mentado en mor de la explicación de estos ambos conceptos precedentemente mencionados, por ello una dilucidación del concepto de valor (como la primera manifestación de toda la formación social), va aparejado a su análisis genético de la concepción temprana de Marx de la formación social en su controversia con sus fuentes contemporáneas, las cuales serán marcadamente influyentes en su teoría del valor.

\section{MÉTODO COMO RECONSTRUCCIÓN DE LA MODERNA SOCIEDAD BURGUESA}

En un diálogo crítico con la dialéctica de dominación y servidumbre propia de la fenomenología hegeliana, es necesario re-pensar la naturaleza de la asociación en el sentido de dominación concreta (desarrollada) del capital en el hombre laboralmente activo ${ }^{25}$ ya que existe una cierta visión «antropologizante» de la ciencia en los primeros trabajos de Marx que abre paso a una crítica empírica de la sociedad en el marco de un determinismo económico «alienante». Por ejemplo, es importante acá recalcar que en el trabajo inicial de Manuscritos económico-filosóficos, se trataba en principio de discutir la socialidad [Gesellschaftlichkeit] de la especie humana en un contexto donde el ser humano aparece en la trama como alienado e instrumentalizado por la división social del trabajo.

Sólo sobre la base de esta disputa podemos entender la continuidad genética en el concepto de formación social en el joven Marx de los Manuscritos de París y el Marx tardío del Capital. La resolución teórica de su concepción

24 «La economía política solo ha analizado, aunque de manera imperfecta, el valor y la medida y ha descubierto el contenido oculto en estas formas. Ésta nunca hizo la pregunta, ¿por qué este contenido toma esa forma? Entonces, ¿por qué el trabajo tiene valor y la medida del trabajo está representada por su duración en el valor del producto de trabajo?» (MEW 23, p.94 f) 25 Cf. Heinrich $1991^{1}$, p.103. 
antropológica, a su vez, estructura el discurso sobre la socialidad y el papel del hombre en su «presentación». Ergo: solo como un ser humano conceptual «deshumanizado»-no antropologizado- puede el sujeto ser teorizado en una representación no empírica. ${ }^{26}$ Una persona concebida de manera abstracta a la que solo se le permite usar la mercancía «en el mercado» no es otra cosa que la mera «re-presentación» de la mercancía. Ya no como un «ser» humano alienado, sino como una forma de manifestación [Erscheinungsform] personificada de todo el modo de producción es que el ser humano podrá ser pensado críticamente. La mercancía que él representa es el verdadero punto de partida de la crítica de la economía política, no el trabajo, no el hombre, y no la historia originaria de la sociedad capitalista. La mercancía (valor) es concebida, por lo tanto, como la verdadera génesis del capital.

Incluso ya desde las primeras líneas contenidas en el corpus del Capital se define todo el propósito de la presentación crítica de la socialización capitalista, se nos advierte de su desarrollo. Por ende, es necesario analizar el modo de producción predominante de la «riqueza» capitalista, en la cual la mercancía aparece como la «forma elemental» de toda la génesis reconstructiva. La totalidad de la sociedad en el desarrollo de la forma valor es la forma de manifestación abstracta de lo que se presenta como riqueza del capital en una determinación más concreta. Solo de un modo dialéctico, aquí solo generalmente determinado, debe y puede entenderse este análisis de la sociedad, es decir no bajo la forma de una definición, sino como un movimiento entre etapas sincrónicas, cada una de las cuales constituye un «momento» metodológico. ${ }^{27} \mathrm{El}$ punto de partida es un momento que abarca todo y que contiene la integridad de la sociedad. En consecuencia, el valor captura de manera integral todas las conexiones humanas del conjunto de

26 En sentido estricto, el modo de abstracción en el Capital muestra categorías no-empíricas (valor, persona, valor agregado, clase, etc.), que se basan en las categorías empíricas (dinero, ganancias, intereses, renta, etc.).

27 Diferenciamos semánticamente la comprensión marxiana y hegeliana de la dialéctica. La dialéctica en el sentido de Marx significa la caracterización escéptica negativa de la estructura de movimiento del método por el cual el propio Marx califica su presentación. Por el contrario, no usamos la dialéctica en el sentido de Hegel para describir una característica de su método, sino que la restringimos aquí solo al opuesto negativo de la dialéctica de señor y siervo. Si queremos caracterizar el método hegeliano como un todo, en una expresión, preferimos el término «especulativo». Siguiendo a Fulda: «Para Hegel, lo dialéctico como lo negativamente razonable es solo uno de varios momentos del método y ni siquiera el primero de ellos. La contradicción, como ingrediente de cada paso en el progreso metódicamente regulado, es solo un momento de este momento: la última fase del desarrollo de una relación entre dos que precede al surgimiento de una nueva unidad. Por el contrario, para Marx la contradicción con lo más interno y la fuente primera de toda dialéctica, la 'dialéctica' se convierte en una expresión legítima para un todo sistemático en el método.» (Fulda 1978, p.192). 
momentos determinantes necesarios de la sociedad. El concepto de valor aparece inmediatamente así como el estar [Dasein] más abstracto de la riqueza social. El camino de la abstracción real progresiva es a través de una interconexión concreta de los momentos determinantes del capital, por lo que la concretización del valor en evolución refleja la concreción progresiva de la riqueza de la sociedad.

La teoría del valor como la construcción fundamental de la clasificación abstractiva de Marx contiene los pasajes filosóficos más ricos para entender orgánicamente la representación crítica: el valor conceptualiza una representación social específicamente moderna ${ }^{28}$

La explicación del valor es quizás el debate más importante en la recepción de Marx surgido a partir desde la publicación de la edición crítica MEGA. El esfuerzo por sistematizar esta teoría ha llevado a diversos autores a adoptar estrategias de análisis que den cuenta de este vínculo progresivo de la batería de momentos que se concatenan hasta dar con la sociedad burguesa. Geert Reuten, por ej., describe la totalidad social de la abstracción de valor bajo la lupa de un metafórico esquema piramidal con el fin de captar sistemáticamente el creciente grado de complejidad del método en tanto punto de partida necesario para la representación dialéctica global. ${ }^{29}$ Su presentación piramidal «heurístico» intenta dar cuenta de las conexiones inherentes de todos los pasos sincrónicos dialécticos en su progresión. La conexión de los momentos sociales se concretaría en orden descendente a partir de un movimiento conceptual dialéctico entre etapas sincrónicas. Cada momento denota así una determinación sintética, la cual vendría a representar la totalidad social.

Los pensadores contemporáneos, que profundizan en el debate sobre el método de presentación unificado y forman el contexto filosófico, entre otros, son Dieter Wolf, Ingo Elbe, Michael Heinrich y Hans Fr. Fulda dan elementos sustanciales del debate en torno a la exposición metacrítica en Marx en la medida que proporcionan (diferentes) argumentos científicos para la unidad metateórica entre «presentación» y «crítica». Podemos interpretar esta tarea como un desarrollo categorial de la sociedad burguesa en la medida que describe una reproducción social dialéctica de los momentos inmanentes de producción de mercancías. La lógica peculiar detrás del capital demuestra que «su presentación es al mismo tiempo una crítica de una ciencia sesgada en esta perversión.» (Heinrich 1991 ${ }^{1}$, p.246).

El carácter científico de la «lógica del capital» metateórico se manifiesta en la concretización conceptual de los factores sociales. A partir del punto de partida

28 Cf. Vargas 2020, p.4140-4158.

29 Cf. Reuten 2014, p.245. 
(valor del producto), el método de presentación crítica tiene como objetivo capturar la forma de apariencia de una existencia o estar [Dasein] inmediato como momento epistemológico, es decir que mienta una estrategia sistemática y orgánica para conceptualizar ese momento particular. En consecuencia, nuestra defensa de la teoría del valor (esencia del capital) como precondición de la reconstrucción del concepto «sociedad» (modernidad) es el resultado de un pensamiento orgánico de categorización abstractiva. La presentación crítica manifiesta, por ende, la insuficiencia epistemológica de la apariencia, que se suprime mediante un acto correctivo en el movimiento dialéctico como concretización conceptual. Esta estructuración conceptual tiene una relación inherente con la autorrepresentación hegeliana del pensamiento y podemos encontrar numerosos puntos de encuentro terminológicos entre Hegel y Marx, pero siempre y cuando tengamos el suficiente cuidado de no simplemente paralelizar los dos métodos de presentación crítica. A pesar de los detallados y completos análisis del capital marxiano y su estrecha relación con la lógica de Hegel, autores como Christopher Arthur y Enrique Dussel tienden a reducir el orden de abstracción social a un esquema de manifestaciones coincidentes. ${ }^{30}$ Dussel, un lector y comentador experto de la obra de Marx, construye las similitudes de la abstracción real de Marx con la red de conceptos derivados del sistema lógico de Hegel por medio de un método de representación isomórfica para mostrar, así, paso a paso la coherencia lógica en el capital. ${ }^{31}$

Sin embargo, esta homología teórica se torna demasiado débil para explicar los puntos de conexión concretos de los momentos isomorfos de ambos sistemas. En un sentido propedéutico, es un medio útil para aclarar las afirmaciones epistemológicas de Marx. Pero, stricto sensu, no logra sortear la arbitrariedad que está a la base de la comparación de esta serie de momentos permaneciendo vagamente relacionados en torno a su epistemológica relacionalidad [Verbindlichkeit]. Sobre todo, este isomorfismo ya no podrá funcionar tan pronto, y en la medida que, la mayoría de las determinaciones económicas históricas en el trabajo tardío de Marx no pueden reducirse, ni traducirse a categorías onto-lógicas.

La reconstrucción socioestructural de la crítica de la economía política se posiciona, en consecuencia, en el llamado «marxismo occidental» como una nueva lectura de Marx en la que se cuestionan los clásicos y ortodoxos paradigmas marxistas que hemos explicado en la primera parte de este artículo.

30 Cf. Dussel 1985, p.10ss, también Lebowitz 2009, p.203. Sobre la discusión entre Tony Smith y Christopher Arthur en torno a esta tesis de homología de Marx-Hegel, cf. Smith 2003, pp.185-194 y su respuesta cf. Arthur 2003, pp.195-198.

31 En ese respecto es Sohn-Rethel (Cf. 2001, p.28) quien primero incorpore la categoría abstracción real para la comprensión conceptual que supone las formas sociales de la dominación abstracta. 
Por lo tanto, la problematización de la naturaleza científica de la presentación global hace plausible una argumentación y justificación epistemológica de algunas teorías marxistas específicas, tales como: teoría de la crisis, teoría de la tendencia de la caída de la tasa de ganancia, teoría del Estado, teoría del mercado mundial, teoría de crédito, teoría de la dependencia, etc.

La investigación empírica será, por supuesto, la que proporcione el material para la presentación y representación de la sociedad burguesa como una totalidad orgánica. Aunque podemos descubrir las contradicciones sociales históricas en la investigación empírica, es solo en representación abstracta en donde la explicación científica está localizada. No es la preocupación principal de la presentación crítica mostrar por qué el capital (en su conjunto) parece histórico, sino cómo la combinación de estas determinaciones produce las mismas tendencias que resultan del análisis inmanente de la mercancía. El análisis produce una modalidad de ida y retorno, produce la viabilidad de una vuelta a las categorías precedentes como resultado una restauración del mundo real en tanto totalidad social.

\section{A MODO DE CIERRE}

Con la publicación de Rosdolsky, como hemos mostrado, se dio el puntapié inicial a un debate detallado sobre las afirmaciones epistemológicas del trabajo tardío, junto con una reinterpretación científica de la importancia de los Grundrisse en la discusión metodológica dando nuevos bríos interpretativos para la lectura sobre el método crítico de Marx en el contexto del modo de abstracción empírico de Ricardo y en compás a un análisis a la Ciencia de la lógica de Hegel.

En los Grundrisse Marx sostuvo que el capital es premisa del modo de producción capitalista y de la sociedad burguesa y en efecto, la lógica del capital, encuentra entre el siglo XV y XVIII las bases para su realización efectiva al producir la disociación del trabajo asalariado respecto al capital a través de las instituciones modernas. Al contrario de las tesis epistemológicas clásicas del marxismo anteriormente descritas, Marx no construye una historia de la acumulación primitiva como corolario del Capital (en los últimos capítulos del tomo I), más bien, como hemos visto, conceptualiza las formas sociales de lo social en tanto modo de socialización. Siguiendo los argumentos planteados en los apartados anteriores, es que hemos consideramos la necesidad de (re) pensar una teoría de la dominación social en Marx a partir de la crítica de las formas sociales que se sostienen en la teoría del valor y que son la fuente de la argumentación trazada tanto en los Grundrisse como en El Capital, y que en su posibilidad persiste la actualidad de la epistemología marxiana tardía.

De la aventura crítica del Marx tardío se concluye su actualidad epistemológica: La realidad en el entramado modernidad y capitalismo ya 
no puede ser derivada desde los propios rostros humanos, más bien, debe ser declinada en funciones económicas, abstractas e impersonales para ser efectiva. ${ }^{32}$ El capital, en el proceso de socialización moderno, al asumir la forma de sujeto, como valor que se autovaloriza, constituyéndose a sí mismo en un carácter autónomo mediante una lógica de valorización que opera como crecimiento de dinero devenido capital, es constituyente formal y modalmente de la modernidad. El carácter trágico de esta conclusión, se expresa cuando las certezas del proyecto moderno culminan en la paradoja inmanente ${ }^{33}$ al proceso ciego de valorización del capital donde no son los seres humanos los que producen las abstracciones que los dominan, son sus acciones, relaciones sociales capitalistas, las cuales se realizan porque no hay alternativa para resolver de otra manera la vida moderna, de esto se trata la crisis de la modernidad tardía que es a la vez la crisis de la modernidad como proyecto emancipador: no es la vida una realización del capitalismo, las relaciones mercantiles ingresan al mundo de la vida cotidiana de manera generalizada con el capitalismo y el capital encuentra en la socialidad moderna el potencial completo de su propia realización.

\section{REFERENCIAS BIBLIOGRÁFICAS}

ALTHUSSER, L 1974:. Éléments d'autocritique. Paris: Hachette. 2014: «L'objet du 'Capital'». Althusser, L. et al., Lire Le Capital. Paris: PUF, pp. 245-418.

ARNDT, A 1985': Karl Marx: Versuch über den Zusammenhang seiner Theorie. Bochum: Germinal.

ARTHUR, C 2003: «Once more on the Homology Thesis: A Response to Smith's Reply». Historical Materialism, 11:1. Leiden/Boston: Brill, pp.195-198 2004: The New Dialectic and Marx's Capital. Leiden/Boston: Brill.

BALIBAR, É 2000: La filosofia de Marx. Bs Aires: Nueva Visión. .2011: Spinoza y la política. Bs. Aires: Prometeo.

BOBBIO, N 1999: «La dialéctica de Marx», Ni con Marx ni contra Marx. México D.F.: Fondo de Cultura Económico.

BRENTEL, H 1989: Soziale Form und ökonomisches Objekt. Studien zum Gegenstand und Methodenverständnis der Kritik der politischen Ökonomie. Opladen: Westdeutscher Verlag.

BRETON, S 1982-83: «Hegel ou Spinoza. Réflexion sur l'enjeu d'une alternative», Cahiers Spinoza, 4, pp.61-87.

32 Cf. Schwarz 2006, p.52.

33 Cf. Vargas 2020b, p.29-30. 
CASANOVA, C 2007: «Aproximaciones al concepto de lo político en Marx-Spinoza». TATIÁN, D (comp.). Spinoza. Tercer Coloquio. Córdoba: Brujas, pp.355-366.

ÇIDAM, V 2012: Die Phänomenologie des Wiedergeistes. Baden-Baden: Nomos.

DUSSEL, E 1985: La producción teórica de Marx. Un comentario a los Grundrisse. Mexico D.F.: Siglo XXI.

ENGELS F 1962a: Herrn Eugen Dührung's Umwälzung der Wissenschaft, MARX, K. \& ENGELS F. Werke, tomo 19. Berlin: Dietz Verlag, pp.32-135. 1962b: Die Entwicklung des Sozialismus von der Utopie zur Wissenschaft, MARX, K. \& ENGELS F. Werke, tomo 19. Berlin: Dietz Verlag, pp.210-228. . (1976) [1895]. «Carta de Engels a Werner Sombart», 11 de marzo de 1895, MARX, K. \& ENGELS F. Obras escogidas, tomo III. Moscú: Progreso. 19749: «La contribución a la crítica de la Economía Política de Karl MarX», Cuadernos Pasado y Presente, 1.

ELBE, I 2010: Marx im Westen. Die neue Marx-Lektüre in der Bundesrepublik seit 1965. Berlin: Akademie.

FERNÁNDEZ BUEY, F 1984: «Los gustos y las opiniones de Karl Marx sobre cuestiones literarias y artísticas», Revista Enrahonar: quaderns de filosofia 9. Barcelona: Universitat Autonoma de Barcelona.

FINK-EITEL H 1987: Dialektik und Sozialethik. Bodenheim: Athenaeum.

FULDA H. F 1978: «Dialektik als Darstellungsmethode im Kapital von Marx», Suomen Filosofisen Yhdistyksen vuoskirja, 37, Helsinki: Ajatus.

GALLAS, A 2005: Rezension zu D. Wolf/ H. Paragenings. Zur Konfusion des Wertbegriffs. Beiträge zur Kapital-Diskussion. Wissenschaftliche Mitteilungen 47, Nr. 261, Hamburg: Argument

GONZÁLEZ VARELA, N 2012: «Karl Marx, lector anómalo de Spinoza», Marx, K. Cuaderno Spinoza. Barcelona: Intervención Cultural/Montesinos.

GUERRERO, D 2011: Sismondi, precursor de Marx. Madrid: Maia.

HEGEL, G.W.F. 1986: Enzyklopädie der philosophischen Wissenschaften, HEGEL, G.W.F. Werke, tomo VIII, Suhrkamp: Frankfurt am Main.

HEINRICH, M 1991': Die Wissenschaft vom Wert. Die Marxsche Kritik der politischen Ökonomie zwischen wissenschaftlicher Revolution und klassischer Tradition. Hamburg: VSA.

2018: Karl Marx und die Geburt der modernen Gesellschaft. Biographie und Wertentwicklung Stuttgart: Schmetterling.

HENNING, C 2005: Philosophie nach Marx. 100 Jahre Marxrezeption und die normative Sozialphilosophie der Gegenwart in der Kritik. Bielefeld: Transcript.

HOFF, J 2009: Marx global. Zur Entwicklung des internationalen Marx-Diskurses seit 1965. Berlin: Akademie.

JAPPE, A 2016: Las aventuras de la mercancía, trad. D. L. Sanromán. Logroño: Pepitas

LEBOWITZ, M 2009: Following Marx. Method, Critique, and Crisis. Historical Materialism Book Series Nr. 64, Leiden/Boston: Brill.

LÖWY, M 1975: «Ciencia y revolución: objetividad y punto de vista de clase en las ciencias sociales», Dialéctica y Revolución., México D.F.: Siglo XXI 
MACHEREY, P 1997: Introduction à l'Étique de Spinoza: La seconde partie - La réalité mentale. Paris: PUF.

MACHEREY, P 2014: Hegel o Spinoza. Bs. Aires: Tinta Limón.

MARX, K 1945: Grundrisse der Kritik der politischen Ökonomie. Frankfurt a.M.: Europäische Verlagsanstalt/ Wien: Europa Verlag. 1972: Das Kapital. Kritik der politischen Ökonomie, II. MARX, K. \& ENGELS F. Werke, tomo 24. Berlin: Dietz Verlag. 2012: Das Kapital. Kritik der politischen Ökonomie, III. MARX, K. \& ENGELS F. Werke, tomo 25. Berlin: Dietz Verlag

MORFINO, V 2014: Plural temporality. Transindividuality and the aleatory between Spinoza and Althusser. Leiden/Boston: Brill.

LANGE, O 1966: Economía Política I. México D.F.: Fondo de Cultura Económico.

PULGAR, P 2017: «Die Kritik der politischen Ökonomie als korrigierende Darstellungsweise. Die Marxsche Übernahme des deutschen metaphysischen Verfahrens.» en ARNDT A, GERHARD M, ZOVKO J, BOWMAN, B. (eds.). Hegel-Jahrbuch 2016, Hegels Antwort auf Kant I. Berlin: De Gruyter, pp. 355359 \& NARVAEZ, A 2020: «Zur Diskussion um die Marxsche kritische Darstellung. Ein Dialog mit der Metaphysikkritik», Dialectus - Revista de Filosofia: Dossiê Conexão Hegel-Marx: Novas Leituras 18, Fortaleza: Universidade Federal do Ceará

REICHELT, H 2000: «Grenzen der dialektischen Darstellungsform - oder Verabschiedung der Dialektik? Einige Anmerkungen zur These von Dieter Riedel», MEGA-Studien, Heft 1. Berlin: Akademie, pp.100-126.

REUTEN, G 2014: «An Outline of the Systematic-Dialectical Method: Scientific and Political Significance», Hegel's Logic and Marx's Capital: A Reexamination. Historical Materialism Book Series, Nr. 64. Leiden/Boston: Brill.

REUTEN, G \& WILLIAMS, M 1989: Valueform and the state. The tendencies of accumulation and the determination of economic policy in capitalist society. London: Routledge.

RIEDEL, D 1997: «Grenzen der dialektischen Darstellungsform», MEGA-Studien, Heft 1, Berlin: Akademie, pp.3-40.

ROSDOLSKY, R 2004 [1968]: Génesis y estructura de El Capital de Marx (estudios sobre los Grundrisse). México D.F.: Siglo XXI

ROSENTAL, M. M 1969: Die dialektische Methode der politischen Ökonomie von Karl Marx. Berlin: Dietz.

RUIZ SANJUÁN C 2014: «La evolución teórica del marxismo: del materialismo histórico a la crítica de la conciencia social», ISEGORÍA, Revista de Filosofía Moral y Política, 50, enero-junio. Madrid.

SACRISTÁN, M 2003: M.A.R.X. Barcelona: El Viejo Topo.

SCHÄFER, M 2018. Bürgerliche Gesellschaft und Staat: Zur Rekonstruktion von Marx'Theorie und Kritik des Staates. Würzburg: Königshausen u. Neumann.

SILVA, L 1971: El estilo literario de Marx. México D.F.: Siglo XXI.

SMITH, T 2003: «On the Homology Thesis». Historical Materialism, 11: 1. Leiden/ Boston: Brill, pp.185-194. 
SOHN-RETHEL, A 2001: Trabajo intelectual y trabajo manual. Crítica de la epistemología. Barcelona: El Viejo Topo.

SCHWARZ, R 2006: «Un pionero brasileño». New Left Rewiew 36, enero-febrero.

VARGAS, R 2020a: «Marx y el retorno de la crítica de la economía política como 'crítica' de las formas sociales de la modernidad», Izquierdas 49. Santiago. . 2020b: «Dominación abstracta y transformación espacio temporal en la lógica del capital». El ejercicio del pensar 3, GT-Herencias y Perspectivas del Marxismo. Bs. Aires: CLACSO.

WOLF, D 2004: Zur Konfusion des Wertbegriffs. Beitrag zur Kapital-Diskussion. Wissenschaftliche Mitteilungen Heft 3. Hamburg: Argument

WOLF, F. O 2006: «Marx' Konzept der ,Grenzen der dialektischen Darstellung'», HOFF, J et al (ed.). Das Kapital neu lesen. Münster: Westfälisches Dampfboot, pp.159-188.

ZELENY, J 1968: Die Wissenschaftslogik bei Marx und 'Das Kapital'. Frankfurt a.M.: Europäische Verlagsanstalt.

Roberto VARgas MuÑoz es Investigador Postdoctoral, PROYECTO FONDECYT POSTDOCTORADO No 3200399 en el Instituto de Filosofía de la Pontificia Universidad Católica de Valparaíso

Líneas de investigación

Critica la economía política, epistemología del espacio social y teoría crítica

Publicaciones recientes

Vargas, R. \& Narváez, A. (2021): «La producción social del espacio. Sobre la actualidad del giro espacial en Henri Lefebvre». En Formación en geografía, sentidos, métodos y conceptos: apuntes para el siglo XXI, Ediciones Universidad Alberto Hurtado, Santiago, 2021

Vargas, R. «Marx y el retorno de la crítica de la economía política como 'crítica' de las formas sociales de la modernidad». Revista Izquierdas, Chile, $N^{\circ}$ 49, julio 2020

Correo electrónico: robertovmu@gmail.com

Pablo Pulgar Moya es Profesor Adjunto en Universidad Católica Silva Henríquez

Lineas de investigación

Filosofía clásica alemana, economía política y teoría crítica

Publicaciones recientes

Pulgar, P. \& Clochec, P. (2020): «Límites y desarrollo del concepto de sociedad en Marx y la herencia terminológica desde Hegel», Hybris Revista de Filosofía, Vol. $11 \mathrm{~N}^{\circ}$ 1, Mayo 2020. 
Pulgar, P. \& Narvaez, A. (2020): «Zur Diskussion um die Marxsche kritische Darstellung. Ein Dialog mit der Metaphysikkritik», Dialectus - Revista de Filosofia: Dossiê Conexão Hegel-Marx: Novas Leituras 18, Fortaleza: Universidade Federal do Ceará.

Correo electrónico: pablocesarpulgarmoya@gmail.com 